\title{
On the Convergence Region of Multi-step Chebyshev-Halley-type Schemes for Solving Equations
}

\author{
Ioannis K. Argyros ${ }^{1}$ and Santhosh George ${ }^{2}$ \\ ${ }^{1}$ Department of Mathematical Sciences, Cameron University, Lawton, OK 73505, USA; \\ e-mail: iargyros@cameron.edu \\ ${ }^{2}$ Department of Mathematical and Computational Sciences, National Institute of Technology Karnataka, \\ Mangalore 575 025, India; e-mail: sgeorge@ nitk.edu.in
}

\begin{abstract}
The aim of this article is to extend the convergence region of certain multi-step Chebyshev-Halley-type schemes for solving Banach space valued nonlinear equations. In particular, we find an at least as small region as the region of the operator involved containing the iterates. This way the majorant functions are tighter than the ones related to the original region, leading to a finer local as well as a semi-local convergence analysis under the same computational effort. Numerical examples complete this article.
\end{abstract}

\section{Introduction}

Let $F: D \subset \mathcal{B}_{1} \rightarrow \mathcal{B}_{2}$ be a twice continuously differentiable operator in the sense of Fréchet, where $\mathcal{B}_{1}, \mathcal{B}_{2}$ are Banach spaces and $D$ is a nonempty and open set. We shall denote by $\mathcal{L}\left(\mathcal{B}_{1}, \mathcal{B}_{2}\right)$ the space of bounded linear operators from $\mathcal{B}_{1}$ into $\mathcal{B}_{2}$.

Numerous problems in mathematical, scientific and engineering computing [1-19] are usually formulated like an equation of the form

$$
F(x)=0 .
$$

Received: February 18, 2019; Accepted: March 16, 2019

2010 Mathematics Subject Classification: 65H10, 47H17, 49M15, 65D10, 65 G99.

Keywords and phrases: Chebyshev method, Halley method, local convergence, semi-local convergence, Fréchet derivative, $\omega$-conditions.

Copyright ( 2019 Ioannis K. Argyros and Santhosh George. This is an open access article distributed under the Creative Commons Attribution License, which permits unrestricted use, distribution, and reproduction in any medium, provided the original work is properly cited. 
However, a solution $x_{*}$ of equation (1.1) can rarely be found in closed form. Therefore, researchers and practitioners resort to iterative schemes to produce a sequence approximating $x_{*}$. Recently, a great effort has been given to generate fast iterative schemes which converge under some Lipschitz-type criteria.

In particular, we consider the Chebyshev-Halley-type scheme defined for each $n=0,1,2, \ldots$, by

$$
\begin{aligned}
& y_{n}=x_{n}-\left[I+\frac{1}{2} M_{n}\left(I-\alpha M_{n}\right)^{-1}\right] F^{\prime}\left(x_{n}\right)^{-1} \\
& z_{n}=y_{n}-F^{\prime}\left(x_{n}\right)^{-1}\left[F^{\prime}\left(x_{n}\right)+F^{\prime \prime}\left(u_{n}\right)\left(x_{n}-y_{n}\right)\right] F^{\prime}\left(x_{n}\right)^{-1} F\left(y_{n}\right) \\
& x_{n+1}=z_{n}-\left[I+M_{n}+\beta M_{n}^{2}\right] F^{\prime}\left(x_{n}\right)^{-1} F\left(z_{n}\right),
\end{aligned}
$$

where $u_{n}=x_{n}-\frac{2}{3} F^{\prime}\left(x_{n}\right)^{-1} F\left(x_{n}\right), M_{n}=F^{\prime}\left(x_{n}\right)^{-1} F^{\prime \prime}\left(u_{n}\right) F^{\prime}\left(x_{n}\right)^{-1} F\left(x_{n}\right), v_{n}=x_{n}-$ $F^{\prime}\left(x_{n}\right)^{-1} F\left(x_{n}\right)$ and $\alpha, \beta \in \mathbb{R}$ (in the local convergence case) and $\alpha \in[0,1]$, $\beta \in[-1,1]$ (in the semi-local convergence case). Iterative schemes-type (1.2) have been considered in [19]. However, in this article, we study the local as well as the semi-local convergence of scheme (1.2) under generalized $\omega$-conditions. Moreover, by introducing the center $\omega$-condition, we locate a subset of $D$ containing the iterates. This subset helps us define tighter majorant functions and parameters than before leading to larger radius of convergence (i.e., we obtain a wider choice of initial guesses); tighter error bounds on the distances $\left\|x_{n+1}-x_{n}\right\|,\left\|x_{n}-x_{*}\right\|$ (i.e., fewer iterates are needed to obtain a desired error tolerance $\varepsilon>0$ ) and an at least as precise information on the location of the solution. Scheme (1.2) is especially useful, when $F^{\prime \prime}$ is a constant. Other favorable cases can be found in [19].

The design of the article is as follows: Section 2 and Section 3 contain the local and semi-local convergence of scheme (1.2), respectively. The numerical examples appear in the concluding Section 4.

\section{Local Convergence Analysis}

We rely on some parameters and scalar functions to show the local convergence 
analysis of scheme (1.2). Let $\gamma_{0}:[0,+\infty) \rightarrow[0,+\infty)$ be a continuous and increasing function with $\gamma_{0}(0)=0$. Suppose that the equation

$$
\gamma_{0}(t)=1
$$

has at least one positive solution. We denote by $\rho_{0}$ the smallest such solution. Let $\gamma:[0,+\infty) \rightarrow[0,+\infty), \lambda_{1}:\left[0, \rho_{0}\right) \rightarrow[0,+\infty), \lambda_{2}:\left[0, \rho_{0}\right) \rightarrow[0,+\infty)$ be continuous and increasing functions with $\gamma(0)=0$. Define functions $\mu_{0}$ and $\mu$ on the interval $\left[0, \rho_{0}\right)$ by

$$
\mu_{0}(t)=\frac{\int_{0}^{1} \gamma((1-\theta) t) d \theta}{1-\gamma_{0}(t)}
$$

and

$$
\mu(t)=\mu_{0}(t)-1
$$

We have $\mu(0)=-1$ and $\mu(t) \rightarrow+\infty$ as $t \rightarrow \rho_{0}^{-}$. The intermediate value theorem assures that equation $\mu(t)=0$ has at least one positive solution. We denote the smallest such solution by $r_{0}$.

Define functions $\mu_{1}, \mu_{2}$ on the interval $\left[0, \rho_{0}\right)$ by

$$
\mu_{1}(t)=\mu_{0}(t)+\frac{\int_{0}^{1} \lambda_{1}(\theta t) d \theta}{3\left(1-\gamma_{0}(t)\right)}
$$

and

$$
\mu_{2}(t)=\mu_{1}(t)-1
$$

Suppose that

$$
\lambda_{1}(0)<3
$$

Then, we get $\mu_{2}(0)<0$ by $(2.2)$ and $\mu_{2}(t) \rightarrow+\infty$ as $t \rightarrow \rho_{0}^{-}$. Denote by $r_{1}$ the smallest positive solution of equation $\mu_{2}(t)=0$. Notice that $r_{1} \leq r_{0}$. Define functions $p$ and $p_{1}$ on $\left[0, \rho_{0}\right)$ by 


$$
p(t)=\frac{\lambda_{2}(t) \int_{0}^{1} \lambda_{1}(\theta t) d \theta t}{\left(1-\gamma_{0}(t)\right)^{2}}
$$

and

$$
p_{1}(t)=|\alpha| p(t)-1
$$

We get $p_{1}(0)=-1$ and $p_{1}(t) \rightarrow+\infty$ as $t \rightarrow \rho_{0}^{-}$. We denote by $\rho_{1}$ the smallest positive solution of equation $p_{1}(t)=0$. Set

$$
\rho=\min \left\{\rho_{0}, \rho_{1}\right\}
$$

Define functions $\mu_{3}, \mu_{4}$ on the interval $[0, \rho)$ by

$$
\mu_{3}(t)=\mu_{0}(t)+\frac{p(t) \int_{0}^{1} \lambda_{1}(\theta t) d \theta}{2(1-|\alpha| p(t))\left(1-\gamma_{0}(t)\right)}
$$

and

$$
\mu_{4}(t)=\mu_{3}(t)-1
$$

We obtain $\mu_{3}(0)=-1$ and $\mu_{3}(t) \rightarrow+\infty$ as $t \rightarrow \rho^{-}$. Denote by $r_{2}$ the smallest positive solution of equation $\mu_{4}(t)=0$. Suppose that equation

$$
\gamma_{0}\left(\mu_{3}(t) t\right)=1
$$

has at least one positive solution. We denote by $\rho_{2}$ the smallest such solution. Define the functions $\mu_{5}, \mu_{6}$ on the interval $\left[0, \rho_{2}\right), \rho_{2}=\min \left\{\rho, \rho_{1}\right\}$ by

$$
\begin{aligned}
\mu_{5}(t)= & \frac{\int_{0}^{1} \gamma\left((1-\theta) \mu_{3}(t) t\right) d \theta \mu_{3}(t)}{1-\gamma_{0}\left(\mu_{3}(t) t\right)} \\
& +\frac{\left(\gamma_{0}\left(\mu_{3}(t) t\right)+\gamma_{0}(t)\right) \int_{0}^{1} \lambda_{1}\left(\theta \mu_{3}(t) t\right) d \theta \mu_{3}(t)}{\left(1-\gamma_{0}\left(\mu_{3}(t) t\right)\right)\left(1-\gamma_{0}(t)\right)}
\end{aligned}
$$




$$
+\frac{\left(1+\mu_{0}(t)\right) \lambda_{2}\left(\mu_{1}(t) t\right) \int_{0}^{1} \lambda_{1}\left(\theta \mu_{3}(t) t\right) d \theta \mu_{3}(t) t}{\left(1-\gamma_{0}(t)\right)^{2}}
$$

and

$$
\mu_{6}(t)=\mu_{5}(t)-1
$$

We have $\mu_{6}(0)=-1$ and $\mu_{6}(t) \rightarrow+\infty$ as $t \rightarrow \rho_{3}^{-}$. Denote by $r_{3}$ the smallest positive solution of equation $\mu_{6}(t)=0$. Suppose that equation

$$
\gamma_{0}\left(\mu_{5}(t) t\right)=1
$$

has at least one positive solution. We denote by $\rho_{3}$ the smallest such solution and set $\rho_{4}=\min \left\{\rho_{2}, \rho_{3}\right\}$. Define functions $\mu_{7}, \mu_{8}$ on the interval $\left[0, \rho_{4}\right)$, by

$$
\begin{aligned}
\mu_{7}(t)= & \mu_{5}(t)+\frac{\left(\gamma_{0}\left(\mu_{5}(t) t\right)+\gamma_{0}(t)\right) \int_{0}^{1} \lambda_{1}\left(\theta \mu_{5}(t) t\right) d \theta \mu_{5}(t)}{\left(1-\gamma_{0}\left(\mu_{5}(t) t\right)\right)\left(1-\gamma_{0}(t)\right)} \\
& +\frac{p(t) \int_{0}^{1} \lambda_{1}\left(\theta \mu_{5}(t) t\right) d \theta \mu_{5}(t)}{1-\gamma_{0}(t)} \\
& +\frac{|\beta| p^{2}(t) \int_{0}^{1} \lambda_{1}\left(\theta \mu_{5}(t) t\right) d \theta \mu_{5}(t) t}{1-\gamma_{0}(t)}
\end{aligned}
$$

and

$$
\mu_{8}(t)=\mu_{7}(t)-1
$$

We get $\mu_{8}(0)=-1$ and $\mu_{8}(t) \rightarrow+\infty$ as $t \rightarrow \rho_{5}$. Denote by $r_{4}$ the smallest positive solution on equation $\mu_{8}(t)=0$. Define radius of convergence $r$ by

$$
r=\min \left\{r_{1}\right\}, \quad i=1,2,3,4 .
$$

Let $U(x, a)=\left\{y \in \mathcal{B}_{1}:\|x-y\|<a\right\}$ and $\bar{U}(x, a)$ be its closure.

The local convergence analysis of method (1.2) is based on the hypotheses $(\mathrm{H})$ : 
$\left(\mathrm{h}_{1}\right) F: D \subset \mathcal{B}_{1} \rightarrow \mathcal{B}_{2}$ is a continuously differentiable operator in the sense of Fréchet and there exists $x_{*} \in D$ such that $F\left(x_{*}\right)=0$ and $F^{\prime}\left(x_{*}\right)^{-1} \in \mathcal{L}\left(\mathcal{B}_{2}, \mathcal{B}_{1}\right)$.

$\left(\mathrm{h}_{2}\right)$ There exists a function $\gamma_{0}:[0,+\infty) \rightarrow[0,+\infty)$ continuous and increasing with $\gamma_{0}(0)=0$ such that for each $x \in D$

$$
\left\|F^{\prime}\left(x_{*}\right)^{-1}\left(F^{\prime}(x)-F^{\prime}\left(x_{*}\right)\right)\right\| \leq \gamma_{0}\left(\left\|x-x_{*}\right\|\right) .
$$

Set $D_{0}=D \cap U\left(x_{*}, \rho_{0}\right)$, where $\rho_{0}$ is given in (2.1).

$\left(h_{3}\right)$ There exist functions $\gamma:\left[0, \rho_{0}\right) \rightarrow[0,+\infty), \quad \lambda_{1}:\left[0, \rho_{0}\right) \rightarrow[0,+\infty)$, $\lambda_{2}:\left[0, \rho_{0}\right) \rightarrow[0,+\infty)$ with $\gamma(0)=0$, continuous and increasing such that for each $x, y \in D_{0}$

$$
\begin{gathered}
\left\|F^{\prime}\left(x_{*}\right)^{-1}\left(F^{\prime}(y)-F^{\prime}(x)\right)\right\| \leq \gamma_{0}(\|y-x\|) \\
\left\|F^{\prime}\left(x_{*}\right)^{-1} F^{\prime}(x)\right\| \leq \lambda_{1}\left(\left\|x-x_{*}\right\|\right)
\end{gathered}
$$

and

$$
\left\|F^{\prime}\left(x_{*}\right)^{-1} F^{\prime \prime}(x)\right\| \leq \lambda_{2}\left(\left\|x-x_{*}\right\|\right) \text {. }
$$

$\left(\mathrm{h}_{4}\right) \bar{U}\left(x_{*}, r\right) \subset D, \rho_{0}, \rho_{1}, \rho_{3}$ given in (2.1), (2.3), (2.4), respectively exist and (2.2) holds.

$\left(\mathrm{h}_{5}\right)$ There exists $r_{*} \geq r$ such that

$$
\int_{0}^{1} \gamma_{0}\left(\theta r_{*}\right) d \theta<1
$$

Set $D_{1}=D \cap \bar{U}\left(x_{*}, r_{*}\right)$.

The aforementioned hypotheses $(\mathrm{H})$ and notation lead to the local convergence result for method (1.2).

Theorem 2.1. Under the hypotheses $(H)$, sequence $\left\{x_{n}\right\}$ generated by scheme (1.2) for $x_{0} \in U\left(x_{*}, r\right)-\left\{x_{*}\right\}$ converges to $x_{*}$ so that

$$
\left\|y_{n}-x_{*}\right\| \leq \mu_{3}\left(\left\|x_{n}-x_{*}\right\|\right)\left\|x_{n}-x_{*}\right\| \leq\left\|x_{n}-x_{*}\right\|<r
$$




$$
\left\|z_{n}-x_{*}\right\| \leq \mu_{5}\left(\left\|x_{n}-x_{*}\right\|\right)\left\|x_{n}-x_{*}\right\| \leq\left\|x_{n}-x_{*}\right\|
$$

and

$$
\left\|x_{n+1}-x_{*}\right\| \leq \mu_{7}\left(\left\|x_{n}-x_{*}\right\|\right)\left\|x_{n}-x_{*}\right\| \leq\left\|x_{n}-x_{*}\right\|,
$$

where functions $\mu_{3}, \mu_{5}, \mu_{7}$ are given previously and $r$ is defined in (2.5). Moreover, $x_{*}$ is the unique solution of equation $F(x)=0$ in $D_{1}$.

Proof. The definition of the convergence radius $r$ guarantees that for each $t \in[0, r)$

$$
\begin{aligned}
& 0 \leq \gamma_{0}(t)<1, \\
& 0 \leq \mu_{0}(t)<1, \\
& 0 \leq \mu_{1}(t)<1, \\
& 0 \leq|\alpha| p(t)<1, \\
& 0 \leq \mu_{3}(t)<1, \\
& 0 \leq \gamma_{0}\left(\mu_{3}(t) t\right)<1, \\
& 0 \leq \mu_{5}(t)<1, \\
& 0 \leq \gamma_{0}\left(\mu_{5}(t) t\right)<1,
\end{aligned}
$$

and

$$
0 \leq \mu_{7}(t)<1 .
$$

The proof is based on the estimates (2.9)-(2.17) and mathematical induction. Let $x \in U\left(x_{*}, r\right)-\left\{x_{*}\right\}$. Using $\left(\mathrm{h}_{1}\right),\left(\mathrm{h}_{2}\right),(2.5)$ and (2.9), we have:

$$
\left\|F^{\prime}\left(x_{*}\right)^{-1}\left(F^{\prime}(x)-F^{\prime}\left(x_{*}\right)\right)\right\| \leq \gamma_{0}\left(\left\|x-x_{*}\right\|\right) \leq \gamma_{0}(r)<1,
$$

which together with the Banach Perturbation Lemma [3-5], imply that $F^{\prime}(x)^{-1} \in$ $\mathcal{L}\left(\mathcal{B}_{2}, \mathcal{B}_{2}\right)$ and

$$
\left\|F^{\prime}(x)^{-1} F^{\prime}\left(x_{*}\right)\right\| \leq \frac{1}{1-\gamma_{0}\left(\left\|x-x_{*}\right\|\right)}
$$


We must also, show $\left(I-\alpha M_{0}\right)^{-1} \in \mathcal{L}\left(\mathcal{B}_{2}, \mathcal{B}_{1}\right)$. By (2.5), (2.12), (2.18) and $\left(\mathrm{h}_{3}\right)$, we get

$$
\begin{aligned}
\left\|\alpha M_{0}\right\| & \leq \frac{|\alpha| \lambda_{2}\left(\left\|x_{0}-x_{*}\right\|\right) \int_{0}^{1} \lambda_{1}\left(\theta\left\|x_{0}-x_{*}\right\|\right) d \theta\left\|x_{0}-x_{*}\right\|}{\left(1-\gamma_{0}\left(\left\|x_{0}-x_{*}\right\|\right)\right)^{2}} \\
& =|\alpha| p\left(\left\|x_{0}-x_{*}\right\|\right) \leq|\alpha| p(r)<1
\end{aligned}
$$

so

$$
\left\|\left(I-\alpha M_{0}\right)^{-1}\right\| \leq \frac{1}{1-|\alpha| p\left(\left\|x_{0}-x_{*}\right\|\right)}
$$

We can write by $\left(\mathrm{h}_{1}\right)$,

$$
F(x)=F(x)-F\left(x_{*}\right)=\int_{0}^{1} F^{\prime}\left(x_{*}+\theta\left(x-x_{*}\right)\right) d \theta\left(x-x_{*}\right) .
$$

Then, by $\left(\mathrm{h}_{3}\right)$ and $(2.20)$

$$
\left\|F^{\prime}\left(x_{*}\right)^{-1} F(x)\right\| \leq \int_{0}^{1} \lambda_{1}\left(\theta\left\|x-x_{*}\right\|\right) d \theta\left\|x-x_{*}\right\| .
$$

In particular, for $x=x_{0}$, since $x_{0} \in U\left(x_{*}, r\right)-\left\{x_{*}\right\}, y_{0}$ is well defined, if $n=0$ by the first substep of scheme (1.2). By (2.5), (2.13), $\left(\mathrm{h}_{1}\right)-\left(\mathrm{h}_{3}\right),(2.18)$, scheme (1.2) for $n=0$, we get in turn that

$$
\begin{aligned}
\left\|y_{0}-x_{*}\right\|= & \left\|x_{0}-x_{*}-F^{\prime}\left(x_{0}\right)^{-1} F\left(x_{0}\right)+\frac{1}{2} M_{0}\left(I-\alpha M_{0}\right)^{-1} F^{\prime}\left(x_{0}\right)^{-1} F\left(x_{0}\right)\right\| \\
\leq & \left\|F^{\prime}\left(x_{0}\right)^{-1} F\left(x_{*}\right)\right\| \int_{0}^{1}\left\|F^{\prime}\left(x_{*}\right)^{-1}\left(F^{\prime}\left(x_{0}+\theta\left(x_{0}-x_{*}\right)\right)-F^{\prime}\left(x_{0}\right)\right)\right\| d \theta \\
& \left\|x_{0}-x_{*}\right\| \\
& +\frac{1}{2}\left\|M_{0}\right\|\left\|\left(I-\alpha M_{0}\right)^{-1}\right\|\left\|F^{\prime}\left(x_{0}\right)^{-1} F^{\prime}\left(x_{*}\right)\right\|\left\|F^{\prime}\left(x_{*}\right)^{-1} F\left(x_{0}\right)\right\| \\
& {\left[\frac{\int_{0}^{1} \gamma\left((1-\theta)\left\|x_{0}-x_{*}\right\|\right) d \theta}{1-\gamma_{0}\left(\left\|x_{0}-x_{*}\right\|\right)}\right.}
\end{aligned}
$$




$$
\begin{gathered}
\left.\frac{p\left(\left\|x_{0}-x_{*}\right\|\right) \int_{0}^{1} \lambda_{1}\left(\theta\left\|x_{0}-x_{*}\right\|\right) d \theta}{2\left(1-|\alpha| p\left(\left\|x_{0}-x_{*}\right\|\right)\right)\left(1-\gamma_{0}\left(\left\|x_{0}-x_{*}\right\|\right)\right)}\right]\left\|x_{0}-x_{*}\right\| \\
=\mu_{3}\left(\left\|x_{0}-x_{*}\right\|\right)\left\|x_{0}-x_{*}\right\| \leq\left\|x_{0}-x_{*}\right\|<r,
\end{gathered}
$$

so (2.6) holds for $n=0$ and $y_{0} \in U\left(x_{*}, r\right)$. By the definition of $r, v_{0}$ and (2.22), $v_{0} \in U\left(x_{*}, r\right)$. Concerning, $u_{0}$, we have in turn as in (2.22)

$$
\begin{aligned}
\left\|u_{0}-x_{*}\right\|= & \left\|\left(x_{0}-x_{*}-F^{\prime}\left(x_{0}\right)^{-1} F\left(x_{0}\right)\right)+\frac{1}{3} F^{\prime}\left(x_{0}\right)^{-1} F\left(x_{0}\right)\right\| \\
\leq & \frac{\int_{0}^{1} \gamma\left((1-\theta)\left\|x_{0}-x_{*}\right\|\right) d \theta\left\|x_{0}-x_{*}\right\|}{1-\gamma_{0}\left(\left\|x_{0}-x_{*}\right\|\right)} \\
& +\frac{1}{3} \frac{\int_{0}^{1} \lambda_{1}\left(\theta\left\|x_{0}-x_{*}\right\|\right) d \theta\left\|x_{0}-x_{*}\right\|}{1-\gamma_{0}\left(\left\|x_{0}-x_{*}\right\|\right)} \\
= & \mu_{1}\left(\left\|x_{0}-x_{*}\right\|\right)\left\|x_{0}-x_{*}\right\| \leq\left\|x_{0}-x_{*}\right\|<r,
\end{aligned}
$$

so $v_{0} \in U\left(x_{*}, r\right)$. Then, by the last condition in $\left(\mathrm{h}_{3}\right)$, we get that

$$
\begin{aligned}
\left\|F^{\prime}\left(x_{*}\right)^{-1} F^{\prime \prime}\left(u_{0}\right)\right\| & \leq \lambda_{3}\left(\left\|u_{0}-x_{*}\right\|\right) \\
& \leq \lambda_{3}\left(\mu_{1}\left(\left\|x_{0}-x_{*}\right\|\right)\left\|x_{0}-x_{*}\right\|\right) .
\end{aligned}
$$

Hence, $z_{0}$ is well defined. Using (2.5), (2.18), (2.14), (2.21), (2.22), (2.23) and second substep of scheme (1.2) for $n=0$ we have in turn that

$$
\begin{aligned}
\left\|z_{0}-x_{*}\right\|= & \|\left(y_{0}-x_{*}-F^{\prime}\left(y_{0}\right)^{-1} F\left(y_{0}\right)\right)+\left(F^{\prime}\left(y_{0}\right)^{-1}-F^{\prime}\left(x_{0}\right)^{-1}\right) F\left(y_{0}\right) \\
& -F^{\prime}\left(x_{0}\right)^{-1} F^{\prime \prime}\left(u_{0}\right)\left[\left(x_{0}-x_{*}\right)+\left(x_{*}-v_{0}\right)\right] F^{\prime}\left(x_{0}\right)^{-1} F\left(y_{0}\right) \| \\
\leq & \frac{\int_{0}^{1} \gamma\left((1-\theta)\left\|y_{0}-x_{*}\right\|\right) d \theta\left\|y_{0}-x_{*}\right\|}{1-\gamma_{0}\left(\left\|y_{0}-x_{*}\right\|\right)}
\end{aligned}
$$




$$
\begin{aligned}
& \leq \frac{\Gamma_{1}}{\left(1-\gamma_{0}\left(\left\|y_{0}-x_{*}\right\|\right)\right)\left(1-\gamma_{0}\left(\left\|x_{0}-x_{*}\right\|\right)\right)}+\frac{\Gamma_{2}}{\left(1-\gamma_{0}\left(\left\|x_{0}-x_{*}\right\|\right)\right)^{2}} \\
& \leq \mu_{5}\left(\left\|x_{0}-x_{*}\right\|\right)\left\|x_{0}-x_{*}\right\| \leq\left\|x_{0}-x_{*}\right\|<r,
\end{aligned}
$$

where

$$
\Gamma_{1}=\left(\gamma_{0}\left(\left\|x_{0}-x_{*}\right\|\right)+\gamma_{0}\left(\left\|y_{0}-x_{*}\right\|\right)\right) \int_{0}^{1} \lambda_{1}\left(\theta\left\|y_{0}-x_{*}\right\|\right) d \theta\left\|y_{0}-x_{*}\right\|
$$

and

$$
\begin{aligned}
\Gamma_{2}=\left(1+\mu_{0}\left(\left\|x_{0}-x_{*}\right\|\right)\right) & \int_{0}^{1} \lambda_{2}\left(\mu_{1}\left(\left\|x_{0}-x_{*}\right\|\right)\left\|x_{0}-x_{*}\right\|\right) \\
& \int_{0}^{1} \lambda_{1}\left(\theta\left\|y_{0}-x_{*}\right\|\right) d \theta\left\|y_{0}-x_{*}\right\|\left\|x_{0}-x_{*}\right\|,
\end{aligned}
$$

so (2.7) holds for $n=0$ and $z_{0} \in U\left(x_{*}, r\right)$. We also have that $x_{1}$ is well defined by the third substep of scheme (1.2) for $n=0$. Next, using (2.5), (2.16), (2.17), (2.18), (2.21), (2.22) and (2.24), we get in turn that

$$
\begin{aligned}
\left\|x_{1}-x_{*}\right\|= & \| z_{0}-x_{*}-F^{\prime}\left(z_{0}\right)^{-1} F\left(z_{0}\right)+\left(F^{\prime}\left(z_{0}\right)^{-1}-F^{\prime}\left(x_{0}\right)^{-1}\right) F\left(z_{0}\right) \\
& -M_{0} F^{\prime}\left(x_{0}\right)^{-1} F\left(z_{0}\right)-\beta M_{0}^{2} F^{\prime}\left(x_{0}\right)^{-1} F\left(z_{0}\right) \| \\
\leq & \mu_{5}\left(\left\|x_{0}-x_{*}\right\|\right)\left\|x_{0}-x_{*}\right\| \\
+ & \frac{\left(\gamma_{0}\left(\left\|z_{0}-x_{*}\right\|\right)+\gamma_{0}\left(\left\|x_{0}-x_{*}\right\|\right)\right) \int_{0}^{1} \lambda_{1}\left(\theta\left\|z_{0}-x_{*}\right\|\right) d \theta\left\|z_{0}-x_{*}\right\|}{\left(1-\gamma_{0}\left(\left\|z_{0}-x_{*}\right\|\right)\right)\left(1-\gamma_{0}\left(\left\|x_{0}-x_{*}\right\|\right)\right)} \\
& +\frac{p\left(\left\|x_{0}-x_{*}\right\|\right) \int_{0}^{1} \lambda_{1}\left(\theta\left\|z_{0}-x_{*}\right\|\right) d \theta\left\|z_{0}-x_{*}\right\|}{1-\gamma_{0}\left(\left\|x_{0}-x_{*}\right\|\right)} \\
& +\frac{|\beta| p^{2}\left(\left\|x_{0}-x_{*}\right\|\right) \int_{0}^{1} \lambda_{1}\left(\theta\left\|z_{0}-x_{*}\right\|\right) d \theta\left\|z_{0}-x_{*}\right\|}{1-\gamma_{0}\left(\left\|x_{0}-x_{*}\right\|\right)}
\end{aligned}
$$




$$
\leq \mu_{7}\left(\left\|x_{0}-x_{*}\right\|\right)\left\|x_{0}-x_{*}\right\| \leq\left\|x_{0}-x_{*}\right\|<r,
$$

so (2.8) holds for $n=0$ and $x_{1} \in U\left(x_{*}, r\right)$.

To finish the induction for estimates (2.6)-(2.8), substitute $x_{0}, y_{0}, z_{0}, v_{0}, u_{0}, x_{1}$ by $x_{k}, y_{k}, z_{k}, v_{k}, u_{k}, x_{k+1}$ in the preceding estimates. Then, from the estimate

$$
\left\|x_{k+1}-x_{*}\right\| \leq c\left\|x_{k}-x_{*}\right\|<r
$$

where $c=\mu_{7}\left(\left\|x_{0}-x_{*}\right\|\right) \in[0,1)$, we conclude that $\lim _{n \rightarrow+\infty} x_{k}=x_{*}$ and $x_{k+1} \in$ $U\left(x_{*}, r\right)$. The uniqueness of the solution part, is shown by letting $G=$ $\int_{0}^{1} F^{\prime}\left(x_{*}+\theta\left(y_{*}-x_{*}\right)\right) d \theta$ for some $y_{*} \in D_{1}$ with $F\left(y_{*}\right)=0$. Then, by $\left(\mathrm{h}_{1}\right),\left(\mathrm{h}_{2}\right)$ and $\left(\mathrm{h}_{5}\right)$ we obtain that

$$
\begin{aligned}
\left\|F^{\prime}\left(x_{*}\right)^{-1}\left(G-F^{\prime}\left(x_{*}\right)\right)\right\| & \leq \int_{0}^{1} \gamma_{0}\left(\theta\left\|x_{*}-y_{*}\right\|\right) d \theta \\
& \leq \int_{0}^{1} \gamma_{0}\left(\theta r_{*}\right) d \theta<1,
\end{aligned}
$$

so $G^{-1} \in \mathcal{L}\left(\mathcal{B}_{2}, \mathcal{B}_{1}\right)$. Finally, in view of the identity

$$
0=F\left(y_{*}\right)-F\left(x_{*}\right)=G\left(y_{*}-x_{*}\right),
$$

we deduce that $x_{*}=y_{*}$.

Remark 2.2. (a) Let $\gamma_{0}(t)=L_{0} t, \gamma(t)=L t$. The radius $\tilde{\rho}_{1}=\frac{2}{2 L_{0}+L}$ was obtained by Argyros as the convergence radius for Newton's method under condition $\left(h_{1}\right)-\left(h_{3}\right)$. Notice that the convergence radius for Newton's method given independently by Rheinboldt [17] and Traub [18] is given by

$$
\tilde{\rho}=\frac{2}{3 L}<\tilde{\rho}_{1}
$$

Let $f(x)=e^{x}-1$. Then $x^{*}=0$. Set $\Omega=U(0,1)$. Then, we have that $L_{0}=e-1<L$ $=e^{1 / L_{0}}$, so $\tilde{\rho}=0.24252961<\tilde{\rho}_{1}=0.3827$. 
Moreover, the new error bounds [3-5] are:

$$
\left\|x_{n+1}-x^{*}\right\| \leq \frac{L}{1-L_{0}\left\|x_{n}-x^{*}\right\|}\left\|x_{n}-x^{*}\right\|^{2},
$$

whereas the old ones $[17,18]$

$$
\left\|x_{n+1}-x^{*}\right\| \leq \frac{L}{1-L\left\|x_{n}-x^{*}\right\|}\left\|x_{n}-x^{*}\right\|^{2}
$$

Clearly, the new error bounds are more precise, if $L_{0}<L$. Clearly, the radius of convergence of method (1.2) given by $\rho^{*}$ is smaller than $\tilde{\rho}_{1}$.

(b) Method (1.2) stays the same if we use the new instead of the old conditions [19]. We can use the computational order of convergence (COC) [3-5]

$$
\xi=\frac{\ln \frac{\left\|x_{n+2}-x_{n+1}\right\|}{\left\|x_{n+1}-x_{n}\right\|}}{\ln \frac{\left\|x_{n+1}-x_{n}\right\|}{\left\|x_{n}-x_{n-1}\right\|}}, \quad \text { for each } n=1,2, \ldots
$$

or the approximate computational order of convergence (ACOC) [3-5]

$$
\xi^{*}=\frac{\ln \frac{\left\|x_{n+2}-x^{*}\right\|}{\left\|x_{n+1}-x^{*}\right\|}}{\ln \frac{\left\|x_{n+1}-x^{*}\right\|}{\left\|x_{n}-x^{*}\right\|}}, \text { for each } n=0,1,2, \ldots
$$

(c) Using (2.6) and

$$
\begin{aligned}
\left\|F^{\prime}\left(x^{*}\right)^{-1} F^{\prime}(x)\right\| & =\left\|F^{\prime}\left(x^{*}\right)^{-1}\left(F^{\prime}(x)-F^{\prime}\left(x^{*}\right)\right)+I\right\| \\
& \leq 1+\left\|F^{\prime}\left(x^{*}\right)^{-1}\left(F^{\prime}(x)-F^{\prime}\left(x^{*}\right)\right)\right\| \\
& \leq 1+q_{0}\left(\left\|x-x^{*}\right\|\right)
\end{aligned}
$$

condition (2.9) can be replaced by

$$
\lambda_{1}(t)=1+\gamma_{0}(t)
$$


or

$$
\lambda_{1}=1+\gamma_{0}\left(\rho_{0}\right)
$$

\section{Semi-local convergence analysis}

We study the semi-local convergence analysis of scheme (1.2) in an analogous way to the local convergence analysis appearing in Section 2. That is why we omit the proofs for which you can also see [19]. The hypotheses on which we base our analysis are (A):

(a $) F: D \subset \mathcal{B}_{1} \rightarrow \mathcal{B}_{2}$ is twice continuously differentiable operator in the sense of Fréchet and there exists $x_{0} \in D, b>0, \eta \geq 0$ such that $F^{\prime}\left(x_{0}\right)^{-1} \in \mathcal{L}\left(\mathcal{B}_{2}, \mathcal{B}_{1}\right)$,

$$
\left\|F^{\prime}\left(x_{0}\right)^{-1}\right\| \leq b
$$

and

$$
\left\|F^{\prime}\left(x_{0}\right)^{-1} F\left(x_{0}\right)\right\| \leq \eta \text {. }
$$

$\left(\mathrm{a}_{2}\right)$ There exists a function $w_{0}:[0,+\infty) \rightarrow[0,+\infty)$ continuous and non-decreasing such that for each $x \in D$

$$
\left\|F^{\prime}(x)-F^{\prime}\left(x_{0}\right)\right\| \leq w_{0}\left(\left\|x-x_{0}\right\|\right),
$$

and equation

$$
w_{0}(t)=1
$$

has at least one positive solution. Denote by $\rho_{0}$ the smallest such solution and set $D_{0}=D \cap U\left(x_{0}, \rho_{0}\right)$. Moreover, suppose that $w_{0}(t) \geq 0$, for each $t>0$, $w_{0}(t \xi) \leq t^{q} w_{0}(\xi)$ for each $t \in[0,1], \xi \in(0,+\infty)$ and $q \in[0,1]$.

(a) There exists function $w:\left[0, \rho_{0}\right) \rightarrow[0,+\infty)$ continuous and non-decreasing such that for each $x, y \in D_{0}$

$$
\left\|F^{\prime}(y)-F^{\prime}(x)\right\| \leq w(\|y-x\|)
$$

where $w(t) \geq 0$, for each $t>0, w(t \xi) \leq t^{q} w(\xi)$ for each $t \in[0,1], \xi \in(0,+\infty)$ and $q \in[0,1]$. 
$\left(\mathrm{a}_{4}\right)$ There exists $K \geq 0$ such that for each $x \in D_{0}$

$$
F^{\prime \prime}(x) \leq K \text {. }
$$

In the literature the following conditions are used instead of $\left(a_{2}\right),\left(a_{3}\right)$ and $\left(a_{4}\right)$.

(a) $)^{\prime}$ There exists function $w_{1}:[0,+\infty) \rightarrow[0,+\infty)$ continuous and non-decreasing such that for each $x, y \in D$

$$
\left\|F^{\prime}(y)-F^{\prime}(x)\right\| \leq w_{1}(\|y-x\|),
$$

where $w_{1}(t) \geq 0$, for each $t>0, w_{1}(t \xi) \leq t^{q} w_{1}(\xi)$ for each $t \in[0,1], \xi \in(0,+\infty)$ and $q \in[0,1]$.

(a) ' There exists $K_{1} \geq 0$ such that for each $x \in D$

$$
F^{\prime \prime}(x) \leq K_{1} \text {. }
$$

Clearly, we have

$$
D_{0} \subseteq D
$$

so for each $t \in\left[0, \rho_{0}\right)$

$$
\begin{gathered}
w_{0}(t) \leq w_{1}(t), \\
w(t) \leq w_{1}(t)
\end{gathered}
$$

and

$$
K \leq K_{1}
$$

Hence, $w, K$ can replace $w_{1}, K_{1}$ in the semi-local convergence of scheme (1.2) and other schemes using the same function $w_{1}$ and parameter $K$. This way, we expand the convergence region, provide tighter error bounds on the distances $\left\|x_{n+1}-x_{n}\right\|$, $\left\|x_{n}-x_{*}\right\|$ and give a more precise information on the location of the solution $x_{*}$. These benefits are obtained under the same computational effort as before, since the computation of $w_{0}, w$ (or $K$ ) as special cases. Notice also that condition $\left(\mathrm{a}_{2}\right)$ helps us define $D_{0}$ and then $w$ (i.e., $w=w\left(w_{0}, D\right)$ ). The set $D_{0}$ contains the iterates $x_{n}$ and by (3.2) is at least as precise as $D$. 
Define

$$
\begin{gathered}
h_{0}(t)=f_{1}(t)+(1+t) f_{2}(t)+\left(1+t+|\beta| t^{2}\right) f_{3}(t), \\
h(t)=\frac{1}{1-h_{0}(t) t}, \\
f(t, u)=\left(\frac{2}{3}\right)^{q} g_{2}\left((t, u) u+t^{2}(1+|\beta|+|\beta| t)\right) g_{2}(t, u) \\
+\frac{1}{q+1}\left(1+t+|\beta| t^{2}\right) g_{2}(t, u) u+\frac{t^{2}\left(1+t+|\beta| t^{2}\right)}{2(1-\alpha t)} g_{2}(t, u) \\
t(1+t) g_{1}(t, u)\left(1+t+|\beta| t^{2}\right) g_{2}(t, u) \\
\frac{t}{2}\left(1+t+|\beta| t^{2}\right)^{2} g_{2}(t, u)^{2},
\end{gathered}
$$

where

$$
\begin{aligned}
& f_{1}(t)=1+\frac{1}{2} \frac{t}{1-\alpha t}, \\
& f_{2}(t)=\frac{t}{2}+\frac{t}{2(1-\alpha t)}+\frac{t^{2}}{2(1-\alpha t)}+\frac{t^{3}}{2(1-\alpha t)^{2}}, \\
& f_{3}(t)=t f_{2}(t)+t(1+t) f_{2}(t)+\frac{t^{2}(1+t)}{2(1-\alpha t)} f_{2}(t)+\frac{t}{2}(1+t)^{2} f_{2}(t)^{2}, \\
& g_{1}(t, u)=\frac{2^{q-1}}{3^{q}} u+\frac{u}{(q+1)(q+2)}+\frac{(1+\alpha) t^{2}}{2(1-\alpha t)}+\frac{t^{3}}{8(1-\alpha t)^{2}}, \\
& g_{2}(t, u)=\left[\left(\frac{2}{3}\right)^{q} u+t^{2}+\frac{(1+t) u}{q+1}+\frac{t^{2}(1+t)}{2(1-\alpha t)}\right] g_{1}(t, u)+\frac{t}{2}(1+t)^{2} g_{1}(t, u)^{2} .
\end{aligned}
$$

Define $g(t)=h_{0}(t) t-1$, notice that $h_{0}(0)=-1<0, \quad h_{0}\left(\frac{1}{2}\right)>0$, so $h_{0}(t)=0$ has at least a root in $\left(0, \frac{1}{2}\right)$. Let $s^{*}$ be the smallest positive root of $h_{0}(t) t-1=0$, then $s^{*}<\frac{1}{2}$ 
We need some auxiliary results.

Lemma 3.1. Let the functions $h_{0}, h, f$ be defined in (3.5), (3.7). Then

(a) $h_{0}(t)$ and $h(t)$ are increasing, $h_{0}(t)>1$ and $h(t)>1$ for $0<t<s^{*}$;

(b) For $t \in\left(0, s^{*}\right)$ and a fixed $u>0, f(t, u)$ is increasing function of $t$, for $u>0$ and a fixed $t \in\left(0, s^{*}\right), f(t, u)$ is increasing function of $u$.

(c) For $0<\alpha<1, t \in\left(0, s^{*}\right)$ and $u>0, f\left(\alpha t, \alpha^{1+q} u\right)<\alpha^{3+3 q} f(t, u)$.

Let $\beta_{0}=b, \eta_{0}=\eta, \alpha_{0}=K b \eta, b_{0}=b \eta w(\eta)$ and $c_{0}=h\left(a_{0}\right) f\left(a_{0}, b_{0}\right)$. Moreover, we define the following sequences

$$
\begin{gathered}
\beta_{m+1}=h\left(a_{m}\right) \beta_{m}, \eta_{m+1}=c_{m} \eta_{m}, \\
a_{m+1}=K \beta_{m+1} \eta_{m+1}, \quad b_{m+1}=\beta_{m+1} \eta_{m+1} w\left(\eta_{m+1}\right), c_{m+1}=h\left(a_{m+1}\right) f\left(a_{m+1}, b_{m+1}\right)
\end{gathered}
$$

where $m \geq 0$. Then it follows that

$$
a_{m+1}=h\left(a_{m}\right) c_{m} a_{m}, \quad b_{m+1} \leq h\left(a_{m}\right) c_{m}^{1+q} b_{m}
$$

Lemma 3.2. Let $s^{*}$ be the smallest positive root of $h_{0}(t) t-1=0$. If

$$
a_{0}<s^{*} \text { and } h\left(a_{0}\right) c_{0}<1
$$

then (a) for $m \geq 0$, it holds that $h\left(a_{m}\right)>0$ and $c_{m}<1$, (b) the sequence $\left\{a_{m}\right\},\left\{b_{m}\right\},\left\{c_{m}\right\}$ and $\left\{\eta_{m}\right\}$ are decreasing, (c) $h_{0}\left(a_{m}\right) a_{m}<1$ and $h\left(a_{m}\right) c_{m}<1$ for $m \geq 0$.

We have the following estimates [19].

(i) $\Gamma_{m+1}=\left[F^{\prime}\left(x_{m+1}\right)\right]^{-1}$ exists and $\left\|\Gamma_{m+1}\right\| \leq h\left(a_{m}\right)\left\|\Gamma_{m}\right\| \leq h\left(a_{m}\right) \beta_{m}=\beta_{m+1}$,

(ii) $\left\|\Gamma_{m+1} F\left(x_{m+1}\right)\right\| \leq h\left(a_{m}\right) f\left(a_{m}, b_{m}\right)\left\|\Gamma_{m} F\left(x_{m}\right)\right\| \leq c_{m} \eta_{m}=\eta_{m+1}$,

(iii) $K\left\|\Gamma_{m}\right\|\left\|\Gamma_{m} F\left(x_{m}\right)\right\| \leq K \beta_{m} \eta_{m}=a_{m}$,

(iv) $\left\|\Gamma_{m}\right\|\left\|\Gamma_{m} F\left(x_{m}\right)\right\| w\left(\left\|\Gamma_{m} F\left(x_{m}\right)\right\|\right) \leq b_{m}$,

(v) $\left\|u_{m}-x_{m}\right\|=\left\|\frac{2}{3} \Gamma_{m} F\left(x_{m}\right)\right\| \leq \frac{2}{3} \eta_{m}$, 


$$
\begin{aligned}
& \text { (vi) }\left\|v_{m}-x_{m}\right\|=\left\|\Gamma_{m} F\left(x_{m}\right)\right\| \leq \eta_{m}, \\
& \text { (vii) }\left\|y_{m}-x_{m}\right\| \leq f_{1}\left(a_{m}\right)\left\|\Gamma_{m} F\left(x_{m}\right)\right\|, \\
& \text { (viii) }\left\|z_{m}-y_{m}\right\| \leq\left[1+a_{m}\right] g_{2}\left(a_{m}\right)\left\|\Gamma_{m} F\left(x_{m}\right)\right\|, \\
& \text { (ix) }\left\|x_{m+1}-x_{m}\right\| \leq h_{0}\left(a_{m}\right)\left\|\Gamma_{m} F\left(x_{m}\right)\right\| \leq h_{0}\left(a_{m}\right) \eta_{m} \text {, where } m \geq 0 .
\end{aligned}
$$

Lemma 3.3. Let the assumptions of Lemma 3.2 and the conditions (A) hold. Then $u_{m}, v_{m}, y_{m}, z_{m}, x_{m+1}$ belong to $U\left(c_{0}, \rho \eta\right)$, where $\rho=\frac{h_{0}\left(a_{0}\right)}{1-c_{0}}$.

Next, we present the main semi-local convergence result for method (1.2) under the hypotheses (A).

Theorem 3.4. Let $F: D \subseteq \mathcal{B}_{1} \rightarrow \mathcal{B}_{2}$ be twice Fréchet differentiable, where $\mathcal{B}_{1}$ and $\mathcal{B}_{2}$ are Banach spaces, $D$ is a non-empty open convex subset. Suppose that $x_{0} \in D$ and hypotheses (A) hold. Let $\bar{U}\left(x_{0}, \rho \eta\right) \subseteq D, \quad a_{0}=K b \eta, \quad b_{0}=b \eta w(\eta)$, $c_{0}=h\left(a_{0}\right) h_{0}\left(a_{0}, b_{0}\right)$ satisfy $a_{0}<s^{*}$ and $h\left(a_{0}\right) c_{0}<1$ where $\rho=\frac{h_{0}\left(a_{0}\right)}{1-c_{0}}$, $s^{*}$ is the smallest positive root of $h_{0}(t) t-1=0$ and $h_{0}, h, f$ are defined previously. Then, starting from $x_{0}$, the sequence $\left\{x_{n}\right\}$ generated by method (1.2) converge to $x_{*}$ with $x_{n}, x_{*} \in U\left(x_{0}, \rho \eta\right)$ and the solution $x_{*}$ of $F(x)=0$ is unique in $U\left(x_{0}, \tilde{\rho} \eta\right) \cap D$, where $\tilde{\rho}=\frac{2}{a_{0}}-\rho$. Furthermore,

$$
\left\|x_{n}-x_{*}\right\| \leq h_{0}\left(a_{0}\right) r_{0}^{n} r^{\frac{(4+3 q)^{n}-1}{3+q}} \frac{1}{1-r_{0} r^{(4+3 q)^{n}}}
$$

where $r_{0}=\frac{1}{h\left(a_{0}\right)}, r=h\left(a_{0}\right) c_{0}$.

\section{Numerical Examples}

The numerical examples are presented in this section. 
Example 4.1. Let $\mathcal{B}_{1}=\mathcal{B}_{2}=\mathbb{R}^{3}, \Omega=\bar{U}(0,1), x_{*}=(0,0,0)^{T}$. Define function $F$ on $\Omega$ for $u=(x, y, z)^{T}$ by

$$
F(u)=\left(e^{x}-1, \frac{e-1}{2} y^{2}+y, z\right)^{T} .
$$

Then, the Fréchet-derivative is given by

$$
F^{\prime}(v)=\left[\begin{array}{ccc}
e^{x} & 0 & 0 \\
0 & (e-1) y+1 & 0 \\
0 & 0 & 1
\end{array}\right]
$$

Notice that using the (2.8)-(2.12), conditions, we get $\gamma_{0}(t)=(e-1) t, \quad \gamma(t)=e^{\frac{1}{e-1}} t$, $\lambda_{1}(t)=\lambda_{2}(t)=e^{\frac{1}{e-1}}$.

Then using the definition of $r_{0}$ and $r$, we have that

$$
\begin{aligned}
& r_{0}=0.14444885915244823348935199192056, \\
& r=0.041513536254307446815570159515119 .
\end{aligned}
$$

Example 4.2. Let $\mathcal{B}_{1}=\mathcal{B}_{2}=C[0,1]$, the space of continuous functions defined on $[0,1]$ and be equipped with the max norm. Let $\Omega=\bar{U}(0,1)$. Define function $F$ on $\Omega$ by

$$
F(\varphi)(x)=\varphi(x)-5 \int_{0}^{1} x \theta \varphi(\theta)^{3} d \theta
$$

We have that

$$
F^{\prime}(\varphi(\xi))(x)=\xi(x)-15 \int_{0}^{1} x \theta \varphi(\theta)^{2} \xi(\theta) d \theta, \text { for each } \xi \in \Omega
$$

Then, we get that $x_{*}=0, \gamma_{0}(t)=7.5 t, \gamma(t)=15 t, \lambda_{1}(t)=\lambda_{2}(t)=15$. This way, we have that

$$
\begin{aligned}
& r_{0}=0.0029787165027481215216764720565834, \\
& r=0.00025772162389070053020029282819792 .
\end{aligned}
$$


Example 4.3. Let us return back to the motivational example. Then, we get that $w_{0}(t)=w(t)=147 t, v(t)=147$. So, we obtain

$$
\begin{aligned}
r_{0} & =0.000002280599520303840650292497016504, \\
r & =0.0000001563215609454908465374514160 .
\end{aligned}
$$

Example 4.4. Let $\mathcal{B}_{1}=\mathcal{B}_{2}=C[0,1], \Omega=\bar{U}\left(x^{*}, 1\right)$ and consider the non-linear integral equation of the mixed Hammerstein-type $[1,2,6-9,12]$ defined by

$$
x(s)=\int_{0}^{1} G(s, t)\left(x(t)^{3 / 2}+\frac{x(t)^{2}}{2}\right) d t,
$$

where the kernel $G$ is the Green's function defined on the interval $[0,1] \times[0,1]$ by

$$
G(s, t)= \begin{cases}(1-s) t, & t \leq s \\ s(1-t), & s \leq t .\end{cases}
$$

The solution $x_{*}(s)=0$ is the same as the solution of equation (1.1), where $F: C[0,1] \rightarrow C[0,1]$ is defined by

$$
F(x)(s)=x(s)-\int_{0}^{1} G(s, t)\left(x(t)^{3 / 2}+\frac{x(t)^{2}}{2}\right) d t,
$$

Notice that

$$
\left\|\int_{0}^{1} G(s, t) d t\right\| \leq \frac{1}{8}
$$

Then, we have that

$$
F^{\prime}(x) y(s)=y(s)-\int_{0}^{1} G(s, t)\left(\frac{2}{3} x(t)^{1 / 2}+x(t)\right) d t,
$$

so since $F^{\prime}\left(x^{*}(s)\right)=I$,

$$
\left\|F^{\prime}\left(x^{*}\right)^{-1}\left(F^{\prime}(x)-F^{\prime}(y)\right)\right\| \leq \frac{1}{8}\left(\frac{3}{2}\|x-y\|^{1 / 2}+\|x-y\|\right) .
$$

Then, we get that $\gamma_{0}(t)=\gamma(t)=\frac{1}{8}\left(\frac{3}{2} t^{1 / 2}+t\right), \lambda_{1}(t)=\lambda_{2}(t)=1+\gamma_{0}(t)$. So, we 
obtain

$$
\begin{aligned}
& r_{0}=0.74068507094596702788891207092092, \\
& r=0.57895531889724227703197811933933
\end{aligned}
$$

\section{References}

[1] G. Alefeld, On the convergence of Halley's method, Amer. Math. Monthly 88 (1981), 530-536.

[2] I. K. Argyros and D. Chen, Results on the Chebyshev method in Banach spaces, Proyecciones 12(2) (1993), 119-128.

[3] I. K. Argyros and A. A. Magreñán, Iterative Methods and Their Dynamics with Applications: A Contemporary Study, CRC Press, 2017.

[4] I. K. Argyros, S. George and N. Thapa, Mathematical Modeling for the Solution of Equations and Systems of Equations with Applications, Volume-I, NY: Nova Publishes, 2018.

[5] I. K. Argyros, S. George and N. Thapa, Mathematical Modeling for the Solution of Equations and Systems of Equations with Applications, Volume-II, NY: Nova Publishes, 2018.

[6] D. D. Bruns and J. E. Bailey, Nonlinear feedback control for operating a non-isothermal CSTR near an unstable steady state, Chem. Eng. Sci. 32 (1977), 257-264.

[7] V. Candela and A. Marquina, Recurrence relations for rational cubic methods I: The Halley method, Computing 44 (1990), 169-184.

[8] V. Candela and A. Marquina, Recurrence relations for rational cubic methods II: The Chebyshev method, Computing 45 (1990), 355-367.

[9] D. Chen, I. K. Argyros and Q. S. Qian, A note on the Halley method in Banach spaces, Appl. Math. Comput. 58 (1993), 215-224.

[10] J. A. Ezquerro and M. A. Hernández, On the R-order of the Halley method, J. Math. Anal. Appl. 303 (2005), 591-601.

[11] M. Ganesh and M. C. Joshi, Numerical solvability of Hammerstein integral equations of mixed type, IMA J. Numer. Anal. 11 (1991), 21-31.

[12] J. M. Gutiérrez and M. A. Hernández, A family of Chebyshev-Halley type methods in Banach spaces, Bull. Austral. Math. Soc. 55(1) (1997), 113-130. 
[13] J. M. Gutiérrez and M. A. Hernández, Recurrence relations for the super-Halley method, Computers Math. Appl. 36 (1998), 1-8.

[14] M. A. Hernández, Reduced recurrence relations for the Chebyshev method, Journal of Optimization Theory and Applications 98 (1998), 385-397.

[15] A. A. Magreñán, Different anomalies in a Jarratt family of iterative root finding methods, Appl. Math. Comput. 233 (2014), 29-38.

[16] A. A. Magreñán, A new tool to study real dynamics: The convergence plane, Appl. Math. Comput. 248 (2014), 29-38.

[17] W. C. Rheinboldt, A unified convergence theory for a class of iterative processes, SIAM J. Numer. Anal. 5 (1968), 42-63.

[18] J. F. Traub, Iterative Methods for the Solution of Equations, AMS Chelsea Publishing, 1982.

[19] X. Wang and J. Kou, Semilocal convergence for a class of improved multi-step Chebyshev-Halley-like methods under extended conditions, Journal of Fixed Point Theory and Applications (2018), to appear. 